\title{
Nephrocalcinosis: Biochemical Evaluation and Genetic Analysis
}

\author{
Menka YAdaV ANd ARVINd BAGGA* \\ Department of Pediatrics, All India Institute of Medical Sciences, New Delhi, India. \\ *arvindbagga@hotmail.com
}

$\mathrm{N}$ ephrocalcinosis, characterized by the deposition of calcium salts in the renal parenchyma, is detected as diffuse renal calcifications on high-resolution ultrasonography or computed tomography. Patients usually do not have symptoms or have features related to the underlying etiology. Rarely, the disease may be severe enough to result in metabolic dysfunction and end-stage renal disease.

The condition may involve either cortical or medullary locations. Cortical nephrocalcinosis is uncommon and results from damage to the renal parenchyma, followed by dystrophic calcification [1]. It is described in chronic glomerulonephritis, cortical necrosis, sickle cell disease, malignancy and trauma. Medullary nephrocalcinosis is much more common and arises from disturbances in calcium homeostasis, mineral reabsorption in the thick ascending limb, or abnormal acid-base regulation in the collecting duct. Medullary nephrocalcinosis is diagnosed in $7-40 \%$ preterm neonates. Chief risk factors include gestation $<32$ weeks and birthweight $<1500 \mathrm{~g}$ [2]. Hypercalciuria might result from acidosis, parenteral nutrition, medications (loop diuretics, vitamin $\mathrm{D}$, corticosteroids, methylxanthines), and high calcium and low phosphorus intake. Nephrocalcinosis resolves by early childhood, while low glomerular filtration rate (GFR) and reduced concentrating capacity may persist [2].

Compared to adults, children often show an underlying metabolic disorder and higher risk of progression, with poor renal function on follow-up [3]. It is, therefore, necessary to identify the underlying cause, initiate therapy if possible, and provide appropriate counseling. Clinical evaluation includes information on prematurity, concomitant diseases, prior therapies, diet, fluid intake, and family history. Careful biochemical evaluation of urine (on two or more occasions) and blood is done to screen for common metabolic disorders [4].

Distal renal tubular acidosis (RTA), varied causes of hypercalciuria, and primary hyperoxaluria $(\mathrm{PH})$ account for most patients; $12.5 \%$ failed to show an etiology [5]. Another retrospective series on 152 German patients showed that the chief causes were idiopathic hypercalciuria $(34 \%)$, primary tubular disorders $(32 \%)$ and vitamin D toxicity (8\%) [6]. Similar findings were reported from other European countries, showing distal RTA, Bartter syndrome, vitamin D toxicity and idiopathic hyper-calciuria in the majority [7].

In the current issue, Ramya, et al. [8] report the etiology in 54 children with nephrocalcinosis managed at a single tertiary care center. Following biochemical studies, the authors report dRTA in $18(33.3 \%)$ and PH in $9(16.7 \%)$ children. There was high frequency of consanguinity $(50 \%)$, and more than a quarter of the patients had positive family history of a similar illness. Standard biochemistry-based definitions were used to define the etiologies; genetic confirmation was sought in only 8 children. A significant proportion had hypercalciuria secondary to RTA, Dent syndrome, Bartter syndrome, and hypomagnesemia. A cause was not found in a minority, suggesting that systematic metabolic evaluation helps in diagnosing the underlying illness in most patients with nephrocalcinosis.

The report has some limitations [8]. First, while the diagnosis of $\mathrm{PH}$ (based on oxalate excretion $>40 \mathrm{mg} / 1.73$ $\mathrm{m}^{2} / \mathrm{d}$ ) was made in 9 patients, genetic studies were done in only four. In the absence of genetic confirmation, the possibility of enteric or dietary hyperoxaluria cannot be excluded [9]. Secondly, the phenotype of $\mathrm{PH}$ associated with mutations in $A G X T, G R H P R$ and HOGAl is variable, and has reasonable implications for management. Therefore, the diagnosis of $\mathrm{PH}$ must be confirmed by enzyme studies or genetic analysis. Patients with specific $A G X T$ mutations, p.Gly170Arg or p.Phe152Ile, respond well to oral pyridoxine [10]. A precise diagnosis is always necessary for patients to be enrolled in clinical trials using silencing RNA [11].

Disorders like distal RTA, Bartter syndrome, Lowe syndrome, Dent disease, and cystinosis have characteristic phenotypes, enabling relatively secure diagnosis. Our 
understanding of the genetic basis of these disorders has considerably improved. Three new genes have been described for distal RTA: WDR72, FOXI1 and ATP6V1C2. Mutations in these genes produce a phenotype similar to that for previously known genes (ATP6VOA4, ATP6V1B1 and $S L C 4 A 1)$ for this disease. Similarly, mutations in multiple genes may result in Bartter syndrome with nephrocalcinosis (SLC12A1, KCNJ1, CLCNKB, CaSR, $M A G E D 2)$, hypomagnesemia with hypercalciuria (CLDN16, CLDN19), Dent disease (CLCN5, OCRL1) and hypophosphatemia with hypercalciuria (SLC34A1, $S L C 34 A 3)$ [3]. A clinical diagnosis is also possible for other diseases, e.g., Fanconi-Bickel syndrome (SLC2A2), 24-hydroxylase deficiency (CYP24A1) and hypercalcemia with hypocalciuria (CaSR, GNA11, AP2S1). However, confirmation of diagnoses by appropriate genetic studies is recommended, before embarking in many instances for specific therapy. A number of conditions including variants in ADCY10, SCL34A1 and CYP24A1 might present with hypercalciuria alone, and misclassified as idiopathic hypercalciuria unless genetic diagnosis is obtained, as might have occurred in the current or previous studies.

Recently, the Hildebrandt group showed that highthroughput screening for 30 genes enables diagnosis in $\sim 15 \%$ patients with unresolved nephrolithiasis/ nephrocalcinosis [12]. The group also reported that in a larger cohort, whole-exome sequencing helped diagnose $\sim 45 \%$ patients with nephrocalcinosis [12]. Higher diagnostic yield was present in the young $(58 \%$ in those $<3$ years), positive family history $(41 \%)$ and consanguinity $(75 \%)$. Given the proportion of subjects with positive family history and consanguinity, the study by Ramya, et al. [8] would have benefited from detailed genetic studies.

Children with nephrocalcinosis should be referred to clinical units experienced in managing such patients. Systematic biochemical screening is recommended for evaluating the underlying cause [4]. Additional genetic diagnosis is useful for confirming the etiology, and counseling parents regarding the likely course of disease and future extrarenal manifestations. It also provides an opportunity for prenatal diagnosis in future pregnancies. While targeted therapies are available for many monogenic disorders, phenotype-genotype correlation will allow patient stratification for future studies. Given the need in most patients to screen for multiple genes, we advise high-throughput genetic testing using the clinical exome approach.

Funding: Nil; Competing interests: None stated.

\section{REFERENCES}

1. Oliveira B, Kleta R, Bockenhauer D, Walsh SB. Genetic, pathophysiological, and clinical aspects of nephrocalcinosis. Am J Physiol Renal Physiol. 2016;311: F1243-52.

2. Schell-Feith EA, Kist-van Holthe JE, van der Heijden AJ. Nephrocalcinosis in preterm neonates. Pediatr Nephrol. 2010;25:221-30.

3. Habbig S, Beck BB, Hoppe B. Nephrocalcinosis and urolithiasis in children. Kidney Int. 2011;80:1278-91.

4. Bagga A, Bajpai A, Menon S. Approach to renal tubular disorders. Indian J Pediatr. 2005;72:771-6.

5. Mantan M, Bagga A, Virdi VS, Menon S, Hari P. Etiology of nephrocalcinosis in northern Indian children. Pediatr Nephrol. 2007;22:829-33.

6. Rönnefarth G, Misselwitz J. Nephrocalcinosis in children: A retrospective survey. Members of the Arbeitsgemeinschaft für Pädiatrische Nephrologie. Pediatr Nephrol. 2000;14:1016-21.

7. Piccoli GB, De Pascale A, Randone O, Vigotti FN, Priola AM, Naretto C, et al. Revisiting nephrocalcinosis: A single-centre perspective. A northern Italian experience. Nephrology (Carlton). 2016;21:97-107.

8. Ramya K, Krishnamurthy S, Sivamurukan P. Etiological profile of nephrocalcinosis in children from Southern India. Indian Pediatr. 2020;57:415-9.

9. Glew RH, Sun Y, Horowitz BL, Konstantinov KN, Barry M, Fair JR, et al. Nephropathy in dietary hyperoxaluria: A potentially preventable acute or chronic kidney disease. World J Nephrol. 2014;3:122-42.

10. Monico CG, Olson JB, Milliner DS. Implications of genotype and enzyme phenotype in pyridoxine response of patients with type I primary hyperoxaluria. Am J Nephrol. 2005;25:183-8.

11. Liebow A, Li X, Racie T, Hettinger J, Bettencourt BR, Najafian $\mathrm{N}$, et al. An investigational RNAi therapeutic targeting glycolate oxidase reduces oxalate production in models of primary hyperoxaluria. J Am Soc Nephrol. 2017;28:494-503.

12. Daga A, Majmundar AJ, Braun DA, Gee HY, Lawson JA, Shril S, et al. Whole exome sequencing frequently detects a monogenic cause in early onset nephrolithiasis and nephrocalcinosis. Kidney Int. 2018;93:204-13. 